\title{
Successful treatment with denosumab in a patient with sacral giant cell tumor of bone refractory to combination therapy with arterial embolization and zoledronic acid: A case report
}

\author{
SHUNJI NISHIMURA ${ }^{1}$, KAZUHIKO HASHIMOTO ${ }^{1}$, AKIHIRO TAN $^{2}$, YUKINOBU YAGYU $^{3}$ and MASAO AKAGI ${ }^{1}$ \\ ${ }^{1}$ Department of Orthopedic Surgery, Kindai University Faculty of Medicine, Osakasayama, \\ Osaka 589-8511; ${ }^{2}$ Department of Orthopedic Surgery, Sakibana Hospital, Izumi, Osaka 594-1105; \\ ${ }^{3}$ Department of Radiology, Kindai University Faculty of Medicine, Osakasayama, Osaka 589-8511, Japan
}

Received June 14, 2016; Accepted August 18, 2016

DOI: $10.3892 / \mathrm{mco} .2017 .1137$

\begin{abstract}
Giant cell tumor of bone (GCTB) is commonly treated with surgery; however, surgery of GCTB in the sacrum may be challenging due to the associated risk. A conservative approach may be selective arterial embolization or zoledronic acid (ZOL) treatment; however, there are currently no studies investigating the efficacy of combining these two treatments. Denosumab may also be used; however, to the best of our knowledge, there are no reports of a stepwise approach for the use of all three treatments in a single patient. We herein present such a case. A 32-year-old woman diagnosed with sacral GCTB was treated with selective arterial embolization for 3 months. No improvement was observed, and monthly infusions of ZOL were added (administered 2 weeks after each arterial embolization treatment). Ten months after the initiation of ZOL, there was still no improvement. The therapy was changed to denosumab $120 \mathrm{mg}$, injected subcutaneously once a month. By the third dose, the buttock pain had decreased and the patient became ambulatory. At 5 and 10 months, computed tomography scans revealed bone sclerosis gradually appearing around the sacrum. By 1 year, needle biopsy detected no neoplastic cells. At that point, the patient discontinued treatment, as there was hepatic function impairment due to a history of hepatitis B. Despite treatment discontinuation, the patient exhibited no further symptoms, there were no signs of progression on radiography, and surgery was not required. Our patient experienced treatment failure
\end{abstract}

Correspondence to: Dr Shunji Nishimura, Department of Orthopedic Surgery, Kindai University Faculty of Medicine, 377-2 Ohno-Higashi, Osakasayama, Osaka 589-8511, Japan E-mail: shunnisi@med.kindai.ac.jp

Abbreviations: CT, computed tomography; GCTB, giant cell tumor of bone; RANK, receptor activator of nuclear factor $\kappa-B$; RANKL, receptor activator of nuclear factor $\kappa-B$ ligand; TRACP-5b, tartrate-resistant acid phosphatase 5b; ZOL, zoledronic acid

Key words: denosumab, sacral giant cell tumor of bone, zoledronic acid, selective arterial embolization, conservative treatment with selective arterial embolization. The combination of ZOL with selective arterial embolization also did not improve the patient's condition. Denosumab was found to be superior to both treatments, achieving tumor remission. The patient remains symptom- and disease-free. Further studies are required, but our results suggest that patients with unresectable GCTB who fail to respond to selective arterial embolization may benefit from denosumab treatment, but not from combination therapy with selective arterial embolization and ZOL.

\section{Introduction}

Giant cell tumor of bone (GCTB) constitutes $~ 5 \%$ of all bone tumors (1). Aggressive GCTB presents as a bulging bone mass. Although treatable with surgical curettage followed by adjuvant therapy, such as liquid nitrogen and phenol, the recurrence rate of GCTB is high (2-4). Since curettage of GCTB in the sacral vertebrae is difficult due to its location, en bloc resection is preferred to prevent recurrence. However, surgery in that site is difficult, and there are issues such as intraoperative bleeding and postoperative dysfunction. Furthermore, the surgical results are not always satisfactory and the recurrence rates for sacral GCTB cannot be reduced through adjuvant therapy. Therefore, conservative treatment must be considered as an option (5).

Although radiotherapy is effective for sacral GCTB, there is a risk of inducing a secondary malignant bone tumor (6). Alternatively, several studies have demonstrated that selective arterial embolization may reduce and control tumors, and is an effective treatment for preserving function (7-9). In addition, infusion of the aminobisphosphonate zoledronic acid (ZOL) reportedly inhibits bone destruction at inoperable sites and prevents recurrence (10-12). While treatments with selective arterial embolism and ZOL are normally performed independently, patients who do not respond to these treatments require other measures.

The anti-receptor activator of nuclear factor $\kappa-\mathrm{B}$ ligand (anti-RANKL) monoclonal antibody, denosumab, was recently released. Denosumab characteristically plays a role in preventing osteolysis by inhibiting the function of osteoclast-like giant cells that are present in GCTB (13-15). In fact, the efficacy of denosumab against GCTB has been evaluated 
A

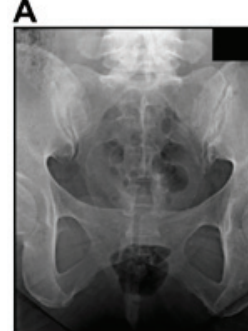

C

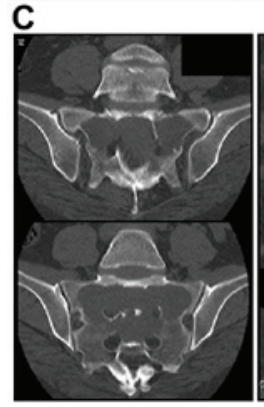

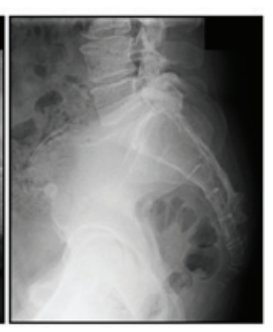
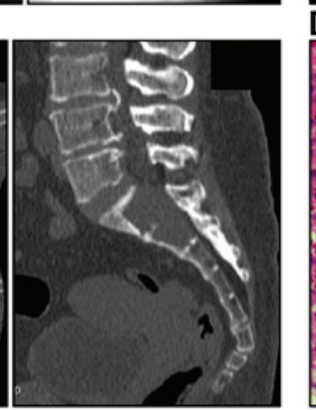

B

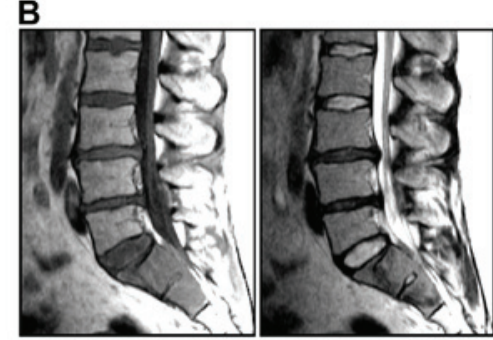

D

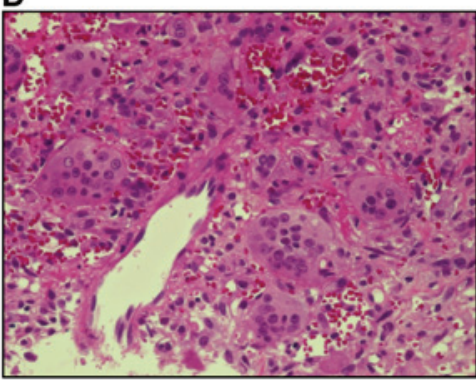

Figure 1. Images and pathological tissues at first visit. (A) Frontal and lateral views of the sacral vertebrae on plain radiography. (B) Magnetic resonance imaging, sagittal plane, T1- and T2-weighted images. Neoplastic lesions are seen in S1-S2. (C) Computed tomography (CT) scan images. Osteolytic images are seen in S1-S2. (D) Histological examination of pathological specimen obtained by a CT-guided core needle biopsy. Numerous stromal cells and osteoclast-like giant cells are concurrently present. Giant cell tumor of bone was diagnosed (hematoxylin and eosin staining; magnification, $\mathrm{x} 50$ ).

in a number of studies $(13,16,17)$; however, there has been no report of a patient receiving all three treatments (selective arterial embolization, ZOL and denosumab) to date.

We herein report a case of a patient with sacral GCTB showing a poor response to combination therapy with arterial embolization and ZOL, who was subsequently treated with denosumab.

\section{Case report}

A 32-year-old woman visited a local clinic with a 5-month history of progressive buttock pain and numbness in the bilateral posterior thighs. The patient had a history of hepatitis B. The physical findings included tenderness in the buttocks and difficulty walking due to persistent buttock pain in the sitting as well as standing positions. Radiography revealed osteolysis in the entire S1-S2 region (Fig. 1A). Magnetic resonance imaging revealed a bulging heterogeneous solid lesion (Fig. 1B). Computed tomography (CT) scans revealed an inner structure without a septal wall and thinning of the surrounding cortical bone (Fig. 1C). The imaging findings were suggestive of an aggressive bone tumor and GCTB was diagnosed by CT-guided bone biopsy (Fig. 1D).

The patient was informed on the risks of surgery and recurrence rates, provided informed consent and opted for conservative therapy. First, selective arterial embolization was performed by a radiologist. The embolization was performed using Gelfoam ${ }^{\circledR}$ (Pfizer Co. New York, NY, USA) once a month for 3 months, but there was no improvement of the clinical symptoms after three procedures, and a hypervascular tumor stain was observed. The response was considered to be poor and intravenous infusion of $4 \mathrm{mg}$ ZOL was initiated concurrently. ZOL was administered once a month, 2 weeks after each arterial embolization. This combination was repeated for 10 months, but the pain did not subside and daily activities were impaired. In addition, CT scans did not reveal distinct bone sclerosis of the sacral cortex (Fig. 2). Therefore, these treatments were discontinued and denosumab was initiated.

The patient received monthly injections of denosumab $120 \mathrm{mg}$ subcutaneously, in addition to daily doses of calcium $610 \mathrm{mg}$, vitamin D $400 \mathrm{IU}$ and magnesium $30 \mathrm{mg}$ orally. The pain had drastically decreased by the third dose of denosumab, and pain on sitting had completely resolved. CT scans taken 5 and 10 months after the initiation of denosumab revealed gradual appearance of bone sclerosis around the sacrum in the transverse and sagittal planes (Fig. 3). Unfortunately, the patient had a history of hepatitis $\mathrm{B}$ and developed hepatic impairment. Hence, denosumab was discontinued after 12 months and treatment for hepatitis was initiated. At that time, a CT-guided needle biopsy was performed to determine the efficacy of our treatment strategy. The tissues displayed remodeled bone formation and a partly fibrous stroma, but no functional stromal cell or osteoclast-like giant component was identified (Fig. 4). The laboratory findings were also noteworthy. The blood levels of tartrate-resistant acid phosphatase 5b (TRACP-5b) were monitored throughout the course of treatment. During combination therapy with arterial embolization and ZOL, the TRACP-5b level was 1,423 mU/dl (normal range, 120-420 mU/dl); by the time denosumab was discontinued, the level had decreased to $233 \mathrm{mU} / \mathrm{dl}$.

Two years after treatment discontinuation, the patient remains symptom-free, shows no radiographic progression of the disease and has not required surgery.

Written informed consent for the publication of this case report and related images was obtained from the patient.

\section{Discussion}

The standard treatment for GCTB is surgery, including adjuvant treatment with liquid nitrogen and ethanol at the time of 
S1
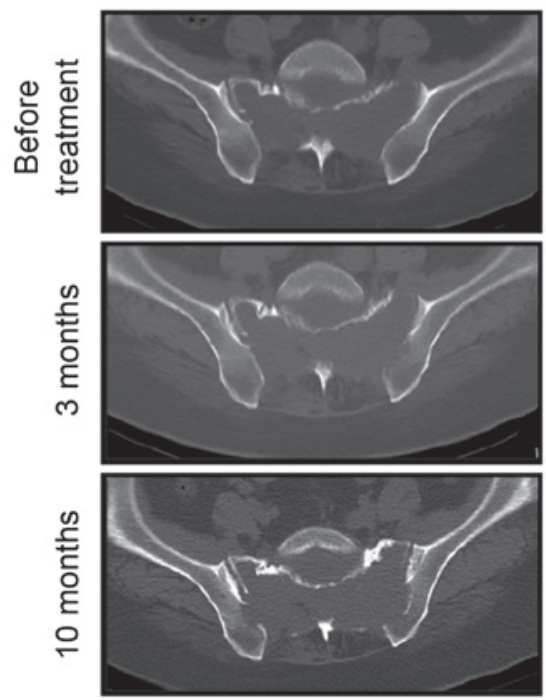

S2
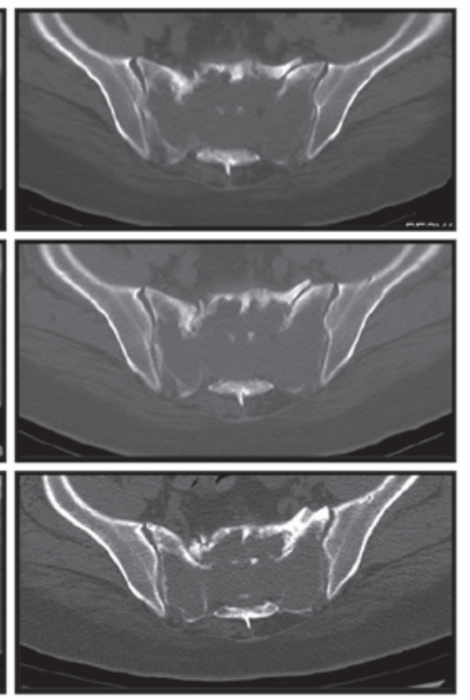

Figure 2. Computed tomography scan images after initiation of treatment with arterial embolization and zoledronic acid. There was a slight change in S1-S2 10 months after the start of treatment compared with prior to treatment, but no shell formation of the bone cortex was observed.
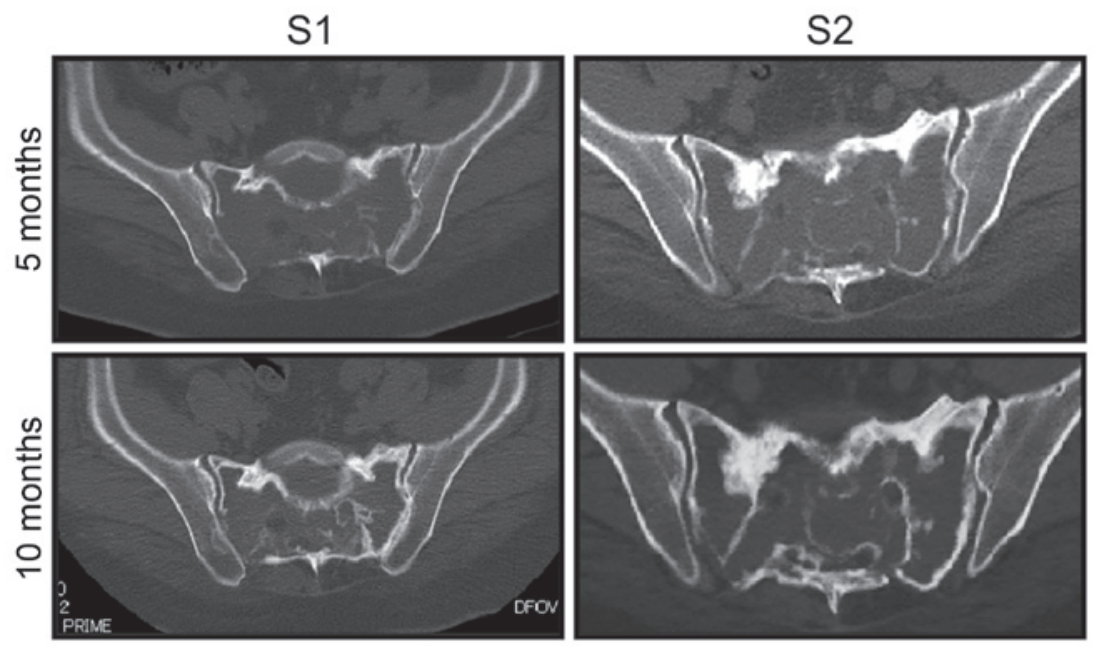

Figure 3. Computed tomography scan images following administration of denosumab. The bone cortex in the anterior sacral vertebrae exhibited thickening 5 months after the start of administration, and shell formation was completely visible at 10 months.

curettage. However, the recurrence rate with or without adjuvant therapy, particularly for GCTB in the sacral vertebrae, is high. Therefore, practitioners must be cautious when deciding on surgical intervention (15). In the present case, conservative therapy was selected to prevent functional impairment.

Arterial embolization and treatment with bisphosphonates are reportedly effective for patients with sacral GCTB. Lin et al (7) reported that tumors were controlled and symptoms improved in 14 of 18 patients, although long-term follow-up to monitor for recurrence was recommended. As in other cases, vascular embolization was performed in our patient. However, the condition did not improve; therefore, ZOL was added to improve efficacy. However, there was no shell formation around the tumor, and angiography revealed a tumor stain. Thus, therapy was switched to denosumab. The TRACP-5b levels, which have recently been shown to be effective for monitoring GCTB (18), began to decrease after the initiation of denosumab

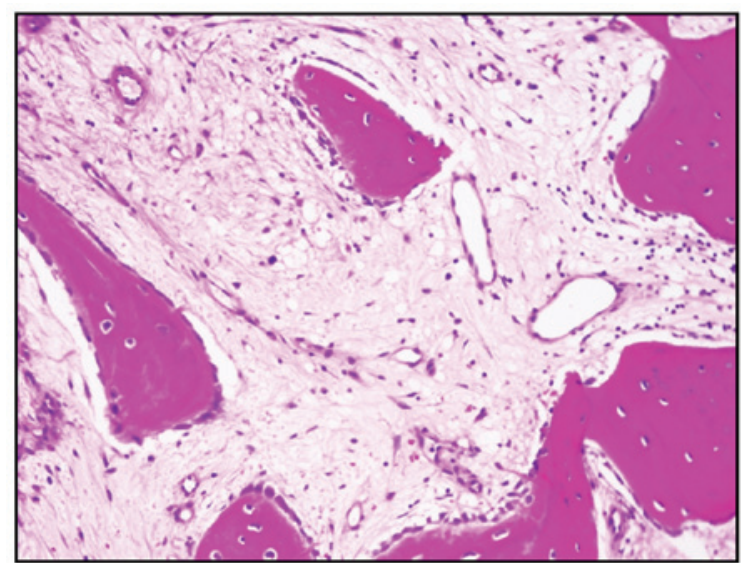

Figure 4. Computed tomography-guided bone biopsy image 1 year after the initiation of treatment with denosumab. New bone tissue has replaced stromal cells and giant cells, demonstrating the efficacy of denosumab (hematoxylin and eosin staining; magnification, $\mathrm{x} 100)$. 
and did not increase after discontinuation of treatment. The tumor did not progress and was deemed stable.

The reasons for our observations may be explained as follows: GCTB consists of osteoclast-like giant cells and oval mononuclear cells (stromal cells). Osteoclast-like giant cells and their precursor cells express receptor activator of nuclear factor $\kappa-\mathrm{B}$ (RANK), whereas oval mononuclear cells express RANK ligand (RANKL) (19), which regulates osteoclast function and plays a role in the formation, function and survival of osteoclasts. Theoretically, bisphosphonates regulate GCTB by reducing the number of osteoclasts and inhibiting osteolysis, and they are reportedly effective for the treatment of GCTB at sites that are difficult to operate (10). For example, a study by Lau et al (20) comparing the antitumor effect of ZOL (a bisphosphonate) with denosumab (another bisphosphonate) on GCTB stromal cells in vitro demonstrated that ZOL acts against tumors in a dose-dependent manner. Furthermore, denosumab has little impact on cell viability, as it does not directly block the RANKL signaling pathway in osteoclast-like giant cells.

By contrast, denosumab was developed as a RANKL-targeted antibody in the field of osteoporosis. Unlike traditional bisphosphonates that induce apoptosis of osteoclasts by adsorbing to the bone surface, denosumab inhibits the formation of osteoclasts and impedes their function and survival (21). Since denosumab is present in body fluids, it enters the haversian canal independent of body surface area, and it may completely inhibit bone remodeling and improve the porosity of cortical bone and the volume of cancellous bone. As such, denosumab has been proven to act on both cortical and cancellous bone (22).

Finally, bone destruction in GCTB is caused by infiltration of osteoclast-like giant cells into the bone. While ZOL exerts antitumor effects on osteoclast-like giant cells through its accumulation in the bone, denosumab is effective in tumors without bone infiltration, due to humoral immunity. Therefore, denosumab may act more extensively on tumors.

To the best of our knowledge, no report has compared the efficacy of ZOL with that of denosumab in a single patient to date. The present case demonstrated the efficacy of denosumab. However, as there have been no long-term studies of patients with unresectable tumors controlled with denosumab, future researchers may wish to investigate when surgery should be added.

In conclusion, we reported a case of a patient responding poorly to combination therapy with arterial embolization and ZOL, a treatment traditionally considered effective for sacral GCTB. Our patient was instead successfully treated with denosumab. To the best of our knowledge, this is the first report of a patient who received arterial embolization, ZOL and denosumab in stages, resulting in a positive clinical response to denosumab.

\section{Acknowledgements}

We would like to thank Honyaku Center Inc. for the English editing of the manuscript.

\section{References}

1. Fletcher CDM, Unni KK and Mertens F: Pathology and genetics of tumours of soft tissue and bone. Lyon, IARC, 2002.
2. Balke M, Ahrens H, Streitbuerger A, Koehler G, Windelmann W, Gosheger G and Hardes J: Treatment options for recurrent giant cell tumors of bone. J Cancer Res Clin Oncol 135: 149-158, 2009.

3. Durr HR, Maier M, Jansson V, Baur A and Refior HJ: Phenol as an adjuvant for local control in the treatment of giant cell tumor of the bone. Eur J Surg Oncol 25: 610-618, 1999.

4. Malawer MM, Bickels J, Meller I, Buch RG, Henshaw RM and Kollender Y: Cryosurgery in the treatment of giant cell tumor. A long term follow-up study. Clin Orthop Relat Res: 176-188, 1999.

5. Marcove RC, Steth DS, Brien EW, Huvos AG and Healey JH: Conservative surgery for giant cell tumors of the sacrum. The role of cryosurgery as a supplement to curettage and partial excision. Cancer 74: 1253-1260, 1994.

6. Shi W, Indelicato DJ, Reith J, Smith KB, Morris CG, Scarborough MT, Gibbs CP Jr, Mendenhall WM and Zlotecki RA: Radiotherapy in the management of giant cell tumor of bone. Am J Clin Oncol 36: 505-508, 2013.

7. Lin PP, Guzel VB, Moura MF, Wallace S, Benjamin RS, Weber KL, Morello FA Jr, Gokaslan ZL and Yasko AW: Long-term follow-up of patients with giant cell tumor of the sacrum treated with selective arterial embolization. Cancer 95: 1317-1325, 2002.

8. Lackman RD, Khoury LD, Esmail A and Donthineni-Rao R: The treatment of sacral giant-cell tumours by serial arterial embolisation. J Bone Joint Surg Br 84: 873-877, 2002.

9. Onishi H, Kaya M, Wada T, Sagoya S, Sasaki M and Yamashita T: Giant cell tumor of the sacrum treatment with selective arterial embolization. Int J Clin Oncol 15: 416-419, 2010.

10. Balke M, Campanacci L, Gebert C, Picci P, Gibbons M, Taylor R, Hogendoorm P, Kroep J, Wass J and Athanasou N: Bisphosphonate treatment of aggressive primary, recurrent and metastatic giant cell tumour of bone. BMC Cancer 10: 462, 2010.

11. Nishisho T, Hanaoka N, Endo K, Takahashi M and Yasui N: Locally administered zoledronic acid therapy for giant cell tumor of bone. Orthopedics 34: e312-e315, 2011.

12. Tse LF, Wong KC, Kumta SM, Huang L, Chow TC and Griffith JF: Bisphosphonates reduce local recurrence in extremity giant cell tumor of bone: A case-control study. Bone 42: 68-73, 2008.

13. Chawla S, Henshw R, Seeger L, Choy E, Blay JY, Ferrari S, Krope J, Grimer R, Reichardt P, Rutkowski P, et al: Safety and efficacy of denosumab for adults and skeletally mature adolescents with giant cell tumour of bone: Interim analysis of an open-label, parallel-group, phase 2 study. Lancet Oncol 14: 901-908, 2013.

14. Raslin KA, Schwab JH, Mankin HJ, Springfield DS and Hornicek FJ: Giant cell tumor of bone. J Am Acad Orthop Surg 21: 118-126, 2013.

15. Ruggieri P, Mavrogenis AF, Ussia G, Angelini A, Papagelopoulos PJ and Mercuri M: Recurrence after and complications associated with adjuvant treatments for sacral giant cell tumor. Clin Orthop Relat Res 468: 2954-2961, 2010.

16. Ueda T, Morioka H, Nishida Y, Kakunaga S, Tsuchiya H, Matsumoto Y, Asami Y, Inoue T and Yoneda T: Objective tumor response to denosumab in patients with giant cell tumor of bone: A multicenter phase II trial. Ann Oncol 26: 2149-2154, 2015.

17. Rutkowski P, Ferrari S, Grimer RJ, Stalley PD, Dijkstra SP, Pienkowski A, Vaz G, Wunder JS, Seeger LL, Feng A, et al: Surgical downstaging in an open-label phase II trial of denosumab in patients with giant cell tumor of bone. Ann Surg Oncol 22: 2860-2868, 2015.

18. Shinozaki T, Saito K, Kobayashi T, Yanagawa T and Takagishi K: Tartrate-resistant acid phosphatase $5 \mathrm{~b}$ is a useful serum marker for diagnosis and recurrence detection of giant cell tumor of bone. Open Orthop J 6: 392-399, 2012.

19. Branstetter DG, Nelson SD, Manivel JC, Blay JY, Chawla S, Thomas DM, Jun S and Jacobs I: Denosumab induces tumor reduction and bone formation in patients with giant-cell tumor of bone. Clin Cancer Res 18: 4415-4424, 2012.

20. Lau CP, Huang L, Wong KC and Kumta SM: Comparison of the anti-tumor effect of denosumab and zoledronic acid on the neoplastic stromal cells of giant cell tumor of bone. Connect Tissue Res 54: 439-449, 2013.

21. Baron R, Ferrari S and Russell RG: Denosumab and bisphosphonates: Different mechanisms of action and effects. Bone 48: 677-692, 2011.

22. Zebaze RM, Libanati C, Austin M, Ghasem-Zadeh A, Hanley DA, Zanchetta JR, Thomas T, Boutroy S, Bogado CE, Bilezikian JP and Seeman E: Differing effects of denosumab and alendronate on cortical and trabecular bone. Bone 59: 173-179, 2014. 